\title{
Study of Buckling Stability on Tall Tower Truss Structure with All Loads
}

\author{
Yixiao Qin ${ }^{1}$, Li Zhang, " , Zhengjun Feng ${ }^{2}$, Chao Zhang ${ }^{3}$ \\ ${ }^{1}$ Mechanical Engineering Institution, Taiyuan University of Science and Technology, Taiyuan, Shanxi, China \\ ${ }^{2}$ Machinery Design and Research Institution, Xuzhou Xu Gong Road Construction Machinery CO., LTD, Tianjin, China \\ ${ }^{3}$ Machinery Design and Research Institution, Hua Dian Heavy Industries CO., LTD, Beijing, China \\ Email address: \\ 1185616066@qq.com (Li Zhang) \\ ${ }^{*}$ Corresponding author
}

\section{To cite this article:}

Yixiao Qin, Li Zhang, Zhengjun Feng, Chao Zhang. Study of Buckling Stability on Tall Tower Truss Structure with All Loads. International Journal of Science, Technology and Society. Vol. 4, No. 4, 2016, pp. 57-62. doi: 10.11648/j.ijsts.20160404.11

Received: May 24, 2016; Accepted: June 5, 2016; Published: June 21, 2016

\begin{abstract}
Tower crane belongs to tall towers truss structures, whose bucking instability often lead to collapse. Past studies focused on the mast instability under a single load, which doesn't apply to the actual condition that tower crane working under various loads at the same time. This paper taking into consideration the tower crane lifting weight as well as various loads like the hoist tipping moment, heavy vertical load, wind load, the centrifugal force, rotary load of resistance and horizontal inertia force. Through the eigenvalue bucking and nonlinear bucking analysis, we can get the lifting load when the hoisting is focusing on a maximum working range of the arm of the tower and tower bar force status and the convergence map of stability. This study provides the complete steps of the tower stability design of tower crane, can be used as a guidance or reference for the actual product design.
\end{abstract}

Keywords: Tower Structure, Buckling Stability, Nonlinearity, Eigenvalue Analysis, Finite Element Method

\section{Introduction}

The truss tower is the main supporting structure of the tower crane, whose loading condition is quite complicated, and analyzing its working condition is sincerely difficult. Under the influence of various loads like the hoist tipping moment, heavy vertical load, wind load, the centrifugal force, rotary load of resistance and horizontal inertia force, it will produce the immense stress and deformation much easier. As a towering truss structure, it's necessary to analyze its stability. With the frequent accidents of tower crane, studying in this area recently attracted the attention of researchers, [1-5] studied the stability problem of two bar truss, [6-9] studied the factors that affect the truss structure of local stability, [10] studied instability of the mast tower crane in static load without wind, under the condition of changing the amplitude of the loading, [11-13] studied the stability of the tower in turbulent winds.

The tower crane in this paper, for instance, with the engineering software ANSYS to simulate the actual operations under the five most dangerous working condition [14-18], our paper conducts linear bucking and nonlinear buckling analysis respectively [19-27], Assuming that the tower crane was working in the most dangerous working condition. Continuously changing the weight and the range of loads to obtain the precise tower crane buckling critical loads, in order to get the convergence map of stability, because the past papers studying in this field was rare and not enough, on one hand, the research what we studying was of significance, on the other hand, It provides useful basis data for tower crane design in order to guarantee the stability of the crane.

\section{Buckling Analysis Model on Tall Tower Truss Structure}

Whether the finite element model is reasonable or not directly affects the accuracy of the analysis results, To make the mast model reasonable, the finite element model should be able to reflect the characteristics of the tower body structure; loading and constraints of the model should be consistent with 
the practical engineering situation. Tower structure entities and loading should be consistent with the real situation. Taking into consideration of the larger structural stiffness at the bottom of the tower, this study assumed fixed bearing, to be able to withstand the bending moment.

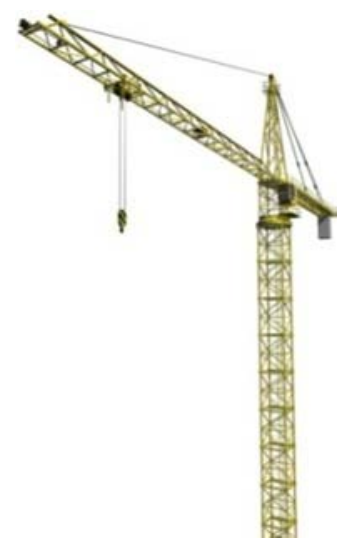

Figure 1. QTZ25 tower crane.

The QTZ25 tower crane is taken as an example to model and conduct buckling analysis. As is shown in Figure 1, the tower crane working range is from $2.5 \mathrm{~m}$ to $35 \mathrm{~m}$, the maximum weight is $2.5 \mathrm{t}$, the nominal weight at the most significant is $0.75 \mathrm{t}$; the main chord is a tube with $100 \mathrm{~mm}$ outer diameter cross-section and $10 \mathrm{~mm}$ thickness; all the abdominal rod are tubes with 100 outer diameter cross-section and 5mmthickness; tower bar material is Q235b, luffing speed $20 \mathrm{~m} / \mathrm{min}$, rotation speed $0.6 \mathrm{r} / \mathrm{min}$, hoisting speed $13.5 / 27 \mathrm{~m} / \mathrm{min}$.

The tower model building process used the command stream. Firstly, we defined the key points on the standard section, connecting points to form the main chord, ventral pole, and divided it into fine mesh. Then we defined the element type and material properties. Considering the structure characteristic and the load of mast tower crane, the BEAM189 space beam is introduced to make the element simulation analysis. This unit is a beam that can bear pulling, pressuring, bending, twisting, furthermore, with the consideration of the shear deformation of Timoshenko beam element, in unit BEAM189 cross-section rotation and torsion can be in the independent interpolation. This unit can also be used with the cross-section defined command, which is a convenient, fast, and accurate way to define the section size. When the tower crane lifting the rated load, the tower main chord should withstand large tensile and compressive stress, so the tower bar material is selected as Q345b, modulus of elasticity $2.1 \times 10^{5} \mathrm{MPa}$, Poisson's ratio is 0.3 , density is 7800 $\mathrm{kg} / \mathrm{m}^{3}$.

Taking into account the characteristics of tower cranes, tower suffers loads, including wind load, weight of the tower, centrifugal force, tipping moment, torque and vertical loads generated by the goods, balance weight and balance boom in the tower body. This paper selects four kinds of potentially most dangerous load condition and performed eigenvalue bucking and nonlinear bucking analysis to mast. What these conditions have in common is that the car is located in the maximum working range of hoists, vertical self weight load remains unchanged, by increasing the goods hoist until it reaches the tower yield limit load on the tower. The differences among the conditions are listed in table 1 and later contents, namely different directions of the wind force, whether to consider various parts of the rotary inertia and centrifugal force, etc. These conditions accurately descript the tower crane stability analysis of the changing of hoisting load and the unchanging rest of the actual load, making the stability analysis reflects the real situation and has practical value. For comparison, the calculation condition commonly used in other documents and results of analysis are listed as below.

The five load conditions are as fellow and shown in Figure 2.

1) The arm located on the cross-sectional diagonal line of tower body does not move, lifting goods, wind direction by the balance of the boom to boom.

2) The arm located on the cross-sectional diagonal line of tower body rotation starting and braking, hanging goods, consistent with the rotary inertia force winds.

3) The tower arm is parallelled to the two sides of the cross-section of the tower does not move, lifting goods, wind direction by the balance of the boom to boom.

4) The tower arm is parallelled to the two sides of the cross-section of the tower rotation starting and braking, hanging rated lifting load, consistent with the rotary inertia force winds.

5) The tower arm located on the cross-sectional diagonal line, there is in no wind, static load conditions, which is commonly used conventional calculation.

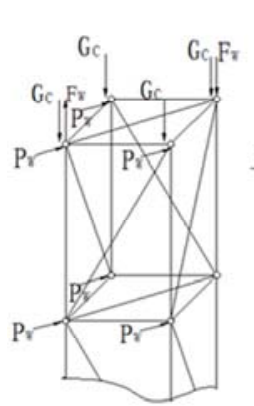

(1)

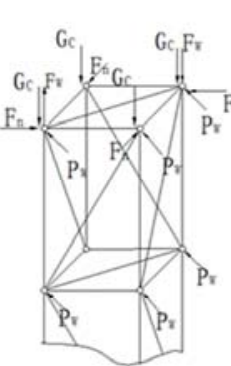

(2)

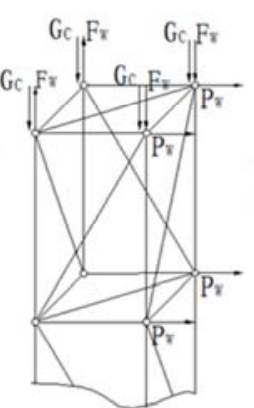

(3)

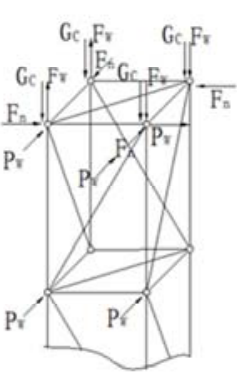

(4)

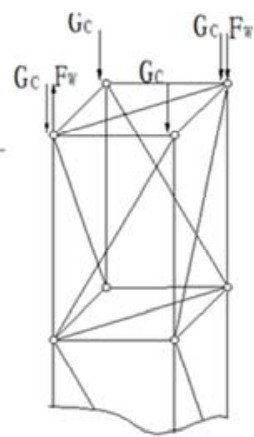

(5)

Figure 2. Different working conditions of the mast equivalent loads. 
Table 1. The calculation condition and the equivalent loads.

\begin{tabular}{llllllll}
\hline \multirow{2}{*}{ Condition $\boldsymbol{i}$} & \multicolumn{6}{l}{ Equivalent loads/N } \\
\cline { 2 - 8 } & $\boldsymbol{G}_{\boldsymbol{c} \mathbf{1}}$ & $\boldsymbol{G}_{\boldsymbol{c} \mathbf{2}}$ & $\boldsymbol{F}_{\boldsymbol{w} \boldsymbol{i}}$ & $\boldsymbol{F}_{\boldsymbol{n}}$ & $\boldsymbol{F}_{\boldsymbol{l}}$ & $\boldsymbol{P}_{\boldsymbol{w} \mathbf{1}}$ & $\boldsymbol{P}_{\boldsymbol{w} \mathbf{2}}$ \\
\hline 1 & $28200^{\uparrow}$ & - & $77000^{\uparrow}$ & - & - & 1056 & - \\
2 & - & $27000^{\uparrow}$ & $33800^{\uparrow}$ & $7800^{\uparrow}$ & 400 & 1056 & - \\
3 & $28200^{\uparrow}$ & - & $54300^{\uparrow}$ & - & - & - & 880 \\
4 & - & $27000^{\uparrow}$ & $24000^{\uparrow}$ & $7800^{\uparrow}$ & 400 & - & 880 \\
5 & - & $27000^{\uparrow}$ & $33800^{\uparrow}$ & - & - & - & - \\
\hline
\end{tabular}

In table 1 , the symbol " $\uparrow$ " indicates that the load increases with the hoist. Good load $G_{h}$, boom weight $G_{b}$, balance weight $G_{p}$, dynamic load coefficient $\phi_{2}$ and dynamic self-weight load coefficient $\phi_{1}$ can get the vertical loads at the top nodes of the tower.

$$
\begin{aligned}
& G_{c 1}=G_{c z} / 4=\left(\phi_{2} G_{h}+\phi_{1} G_{b}+\phi_{1} G_{p}\right) / 4 \\
& G_{c 2}=G_{c z} / 4=\left(G_{h}+G_{b}+G_{p}\right) / 4
\end{aligned}
$$

The goods, the weight of crane trolley, the arm center of gravity, balance arm focus and arm frame beat face form to rotary center distance of heart are respectively $l_{h}, l_{d}, l_{p}, l_{f}$ and the tower width $a$, by the cargo load $G_{h}$, the tower crane boom weight $G_{d}$, weight balance boom weigh $G_{p b}$ and crane counter weight $G_{p}$ can solve the equivalent force of bending.

$$
\begin{aligned}
& F_{w 1}=M_{e 1} / \sqrt{2} a=\left(\phi_{2} G_{h} \cdot l_{h}+\phi_{1} G_{d} \cdot l_{d}-\phi_{1} G_{p d} \cdot l_{p b}\right. \\
& \left.-\phi_{1} G_{p} \cdot l_{p}\right) / \sqrt{2} a \\
& F_{w 2}=M_{e 2} / \sqrt{2} a=\left(G_{h} \cdot l_{h}+G_{d} \cdot l_{d}-G_{p d} \cdot l_{p b}\right. \\
& \left.-G_{p} \cdot l_{p}\right) / \sqrt{2} a \\
& F_{w 3}=M_{e 3} / \sqrt{2} a=\left(\phi_{2} G_{h} \cdot l_{h}+\phi_{1} G_{d} \cdot l_{d}-\phi_{1} G_{p d} \cdot l_{p b}\right. \\
& \left.-\phi_{1} G_{p} \cdot l_{p}\right) / 2 a \\
& F_{w 4}=M_{e 4} / 2 a=\left(G_{h} \cdot l_{h}+G_{d} \cdot l_{d}-G_{p d} \cdot l_{p b}\right. \\
& \left.-G_{p} \cdot l_{p}\right) / 2 a \\
& F_{w 5}=F_{w 2}
\end{aligned}
$$

By turning the start-stop institutions caused the tangential inertial force of goods $P_{\mathrm{CHb}_{h}}$, the tangential inertial force of boom $P_{c H b_{d}}$, the tangential inertial force of balance arm frame $P_{c H b_{p}}$, and arm frame of wind load $P_{w}$, generates equivalent force of torque $T_{n}$.

$$
\begin{aligned}
& F_{n}=T_{n} / 2 a=\left(P_{c H b_{h}} \cdot l_{h}+P_{c H b_{d}} \cdot l_{d}+P_{c H b_{p b}} \cdot l_{p}\right. \\
& \left.+P_{w} \cdot l_{f}\right) / 2 a
\end{aligned}
$$

From the above components quality $m_{i}$, crane rotary angular velocity $\omega$ and centroid to the level of the rotary center distance $\gamma_{i}$, centrifugal force can be obtained.

$$
G_{h} F_{L}=\sum_{i} F_{L i}
$$

The wind pressure of working status $P_{\Pi}$, the windward area $A$ and wind factor $K_{c}$, led to the tower wind load of each standard section.

$$
P_{w 1}=1.2 K_{c} P_{\Pi} A, P_{w 2}=K_{c} P_{\Pi} A
$$

With QTZ25 tower crane parameters in above all, the equivalent load data are shown in table 1.

\section{Mast Tower Crane Stability Analysis}

\subsection{Eigenvalue Buckling Analysis}

The structure under certain load will be in a stable equilibrium state, when the load reaches a certain value, with a small increment, the equilibrium will be broken; the structure will go from original equilibrium state, through the unstable equilibrium state and reaches a new stable equilibrium state. This process is the instability or buckling, and the corresponding load is called the buckling load or critical load.

The element stiffness matrix that is only determined by the stress state is called the geometric stiffness matrix, denoted as $K_{\sigma}$. For any known initial stress state, after a solving for the geometric stiffness matrix $K_{\sigma}$, with application of the load factor $\lambda$ to change initial stress, geometric stiffness matrix changes to $\lambda K_{\sigma}$. Under linear conditions, geometric stiffness matrix $K_{\sigma}$ and stiffness matrix $K^{e}$ are not a displacement function, the equilibrium equation is,

$$
\left(K^{e}+\lambda K_{\sigma}\right) \delta^{*}=P^{*}
$$

Assuming a critical state has been reached, then there exists a perturbations of configuration $\Delta \delta^{*}$ near its shape $\delta^{*}$, so that the system is in equilibrium state under the same external force, then,

$$
\left(K^{e}+\lambda \mathrm{K}_{\sigma}\right)\left(\delta^{*}+\Delta \delta^{*}\right)=P^{*}
$$

Subtracting the above two equations, 


$$
\left(K^{e}+\lambda \mathrm{K}_{\sigma}\right) \Delta \delta^{*}=0
$$

At this point, the eigenvalue buckling analysis is to solve the generalized eigenvalue, namely solving the generalized eigenvalue $\lambda$ and the displacement eigenvectors $\Delta \delta^{*}$. Eigenvalue $\lambda$ times outside-of-structure load gets bifurcation point critical load $P_{c r}$, without considering balance arm frame tower deformation condition, through the constantly adjusting goods load to solve the $P_{c r}$. When the resulting eigenvalue $\lambda$ does not equal to 1 , continue to adjust goods load, until the eigenvalue $\lambda$ was equaled to 1 . ANSYS analysis and calculation results as is shown in table 2.

Table 2. Calculation results.

\begin{tabular}{llllll}
\hline Working condition & $\mathbf{1}$ & $\mathbf{2}$ & $\mathbf{3}$ & $\mathbf{4}$ & $\mathbf{5}$ \\
\hline Buckling load/kN & 100 & 170 & 130 & 210 & 190 \\
Eigenvalue & 0.98951 & 1.00007 & 0.9995 & 0.9995 & 1.002 \\
\hline
\end{tabular}

Shown in the above five kinds of load cases, case 1 eigenvalue buckling load is the minimum, but the most vulnerable to instability. The linear buckling analysis characteristic value of minimum buckling load $100 \mathrm{~N}$ is 10 times more than dynamic allowed maximum lifting load $7.5 \phi_{2} \mathrm{kN}$.

Eigenvalue buckling analysis is a linear analysis, applied to predict the elastic buckling load of the structure under ideal conditions, but the initial defects of the actual structure and the deformation before buckling of the structure will make the load experiencing bucking instability before it reached the theoretical buckling load. Therefore, the critical value of the eigenvalue buckling analysis is usually greater than the non-linear analysis results.

\subsection{Nonlinear Buckling Analysis}

The nonlinear analysis and calculation applied the bilinear isotropic hardening BISO model and the isotropic strengthening Von Mises yield criterion, while opening the large deformation, using the load increment method to progressively load, the arc-length method to calculate in the iterative process, with default convergence conditions. ANSYS analysis reports buckling convergence Figure, convergent Figure's horizontal axis is cumulative iteration number, the vertical axis is absolute convergence norm. Convergence control is associated with L2 norm: L2 value is constantly changing during the calculation; if the L2 is not greater than the tolerance CRIT, then converge.

Considering the geometric and material nonlinearity, material nonlinearity is expressed through two continuous linear, also known as bilinear stress-strain relations. Rod material buckling strength $\sigma_{s} 235=M P a$. For all load cases in Table 1, with hoists of the car located in the maximum working range, ANSYS worked out the non-linear convergence of tower critical loads under five dangerous load conditions are respectively $40 \mathrm{kN}, 64 \mathrm{kN}, 50 \mathrm{kN}, 81 \mathrm{kN}, 68 \mathrm{kN}$. Case 1 has the minimum critical load. As the load gradually adds to $40 \mathrm{kN}$, reaching the material buckling strength, further increment of load results in convergence map does not converge and the deformation of the tower crane. ANSYS analysis does not provide corresponding stress cloud figure. Mast tower crane nonlinear convergence map and stress cloud shown in Figure 3.
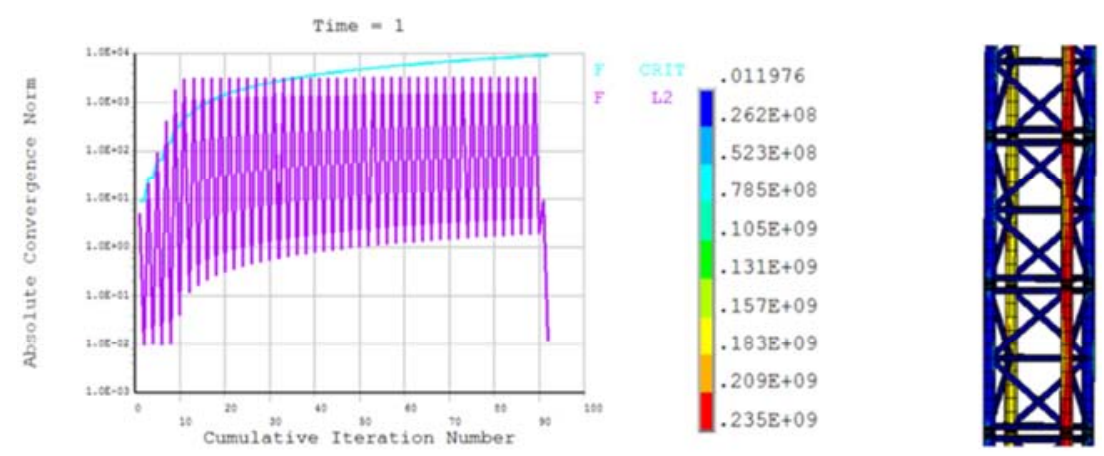

(1)
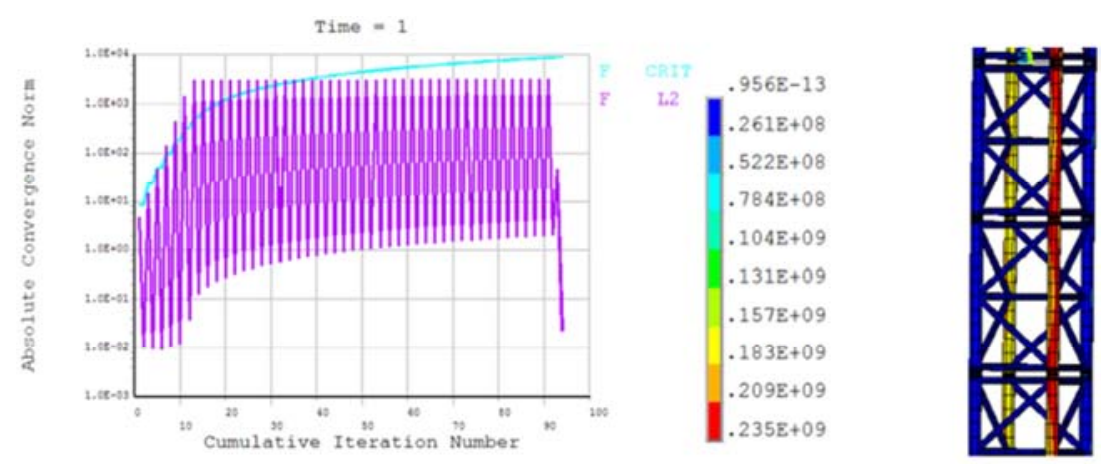

(2) 

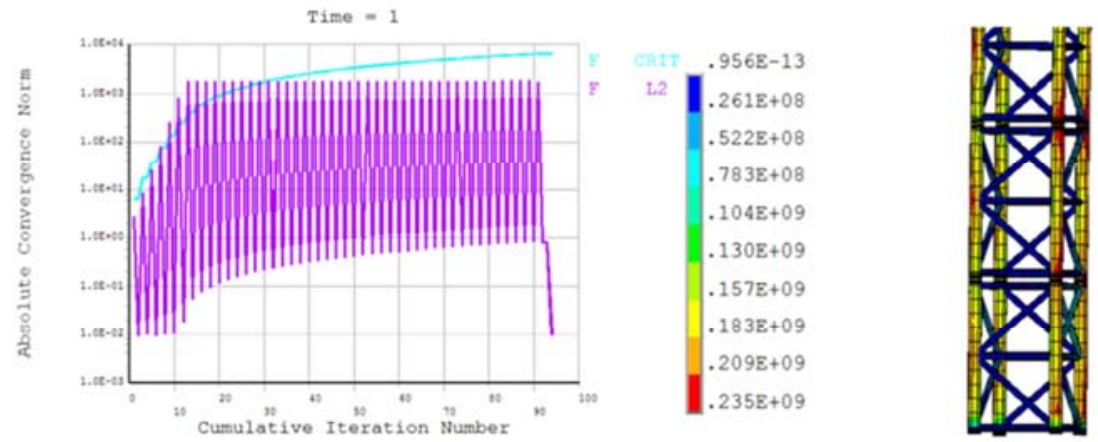

(3)
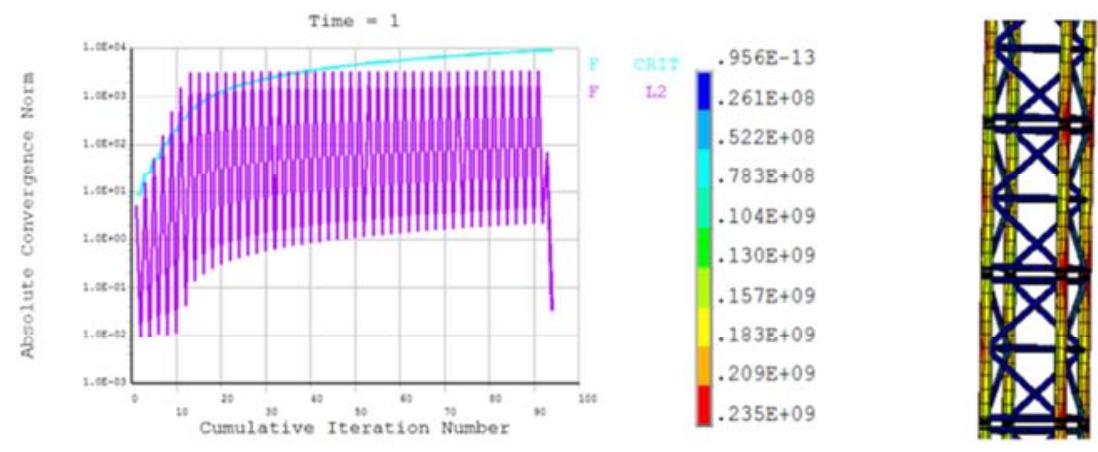

(4)
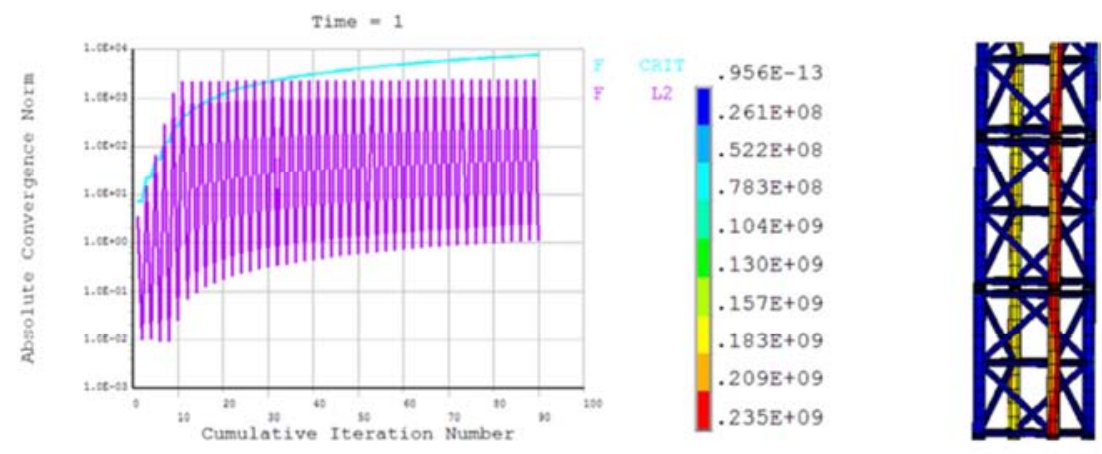

(5)

Figure 3. Diagrams of nonlinear convergence and the tower stress cloud.

Table 3. Mast tower crane nonlinear convergence and stress conditions.

\begin{tabular}{llllll}
\hline Condition & $\mathbf{1}$ & $\mathbf{2}$ & $\mathbf{3}$ & $\mathbf{4}$ & $\mathbf{5}$ \\
\hline Bucking critical load/kN & 40 & 64 & 52 & 84 & 68 \\
Stress/MPa & 235 & 235 & 235 & 235 & 235 \\
\hline
\end{tabular}

From table 3, case 1 has tower bucking critical load as $40 \mathrm{kN}$, 4.2 times the maximum allowed dynamic lifting loads $7.5 \phi_{2}$ $\mathrm{kN}$, but less than the eigenvalue buckling analysis result 100 $\mathrm{KN}$, which means the result of the nonlinear analysis value is less than the results of linear characteristic value, the linear characteristic value of critical load can't be used as the buckling analysis as final result.

\section{Conclusions}

(1) This paper is focusing on the working properties of tower crane, using the load conditions of design specifications and considering all loads, with an increasing hoist load, analyzing the characteristics of tower crane, linear eigenvalue buckling and material nonlinear buckling on the high-rise tower truss structure based on the space frame model. The results show that chord is most likely to experience instability, while the critical load from nonlinear buckling analysis is much smaller than the critical load from eigenvalue buckling analysis.

(2) In this paper, the study of mast of tower cranes is done by eigenvalue buckling and nonlinear buckling analysis, where in, eigenvalue buckling analysis the material nonlinearity, initial defects and deformation before buckling are often not included, thus the resulting buckling load is an ideal load, instead of a practical load accurately reflecting the real situation of mast buckling, taking into consideration the structure stability plasticity behavior and the significant deformation when doing the 
mast nonlinear buckling analysis. Therefore, the nonlinear buckling analysis is necessary and important.

\section{Funding}

The authors thank the National Natural Science Foundation of China (Grant No. 51478290) and the Natural Science Foundation of Shanxi Province, China (Grant No. 2013011022-6) for their support of this research.

\section{References}

[1] M. Ohsaki, J. Y. Zhang, Nonlinear programming approach to form-finding and folding analysis of tensegrity structures using fictitious material properties, 69-70 (2015) 1-10

[2] Pi Yonglin, Mark Andrew Bradford, Non-linear buckling and post buckling analysis of arches with unequal rotational end restraints under a central concentrated load, 49 (2012) 3762-3773

[3] Zhang LW, Lei ZX, Liew ZX, Liew KM. Vibration characteristic of moderately thick functionally graded carbon nanotube reinforced composite skew plates. Compos Struct 2015; 122: 172-83

[4] Lei ZX, Zhang LW, Liew KM. Dynamic stability analysis of carbon nanotube-reinforce functionally graded cylindrical panels using the element-free kpritz method. Compos Struct 2014; 113: 328-338

[5] Zhang LW, Lei ZX, Liew KM. Buckling analysis of FG-CNT reinforced composite thick skew plates using an element-free opproach. Compos Part B: Eng 2015; 54: 36-46

[6] Weng YJ, Cheng YM. Analyzing variable coefficient advection-diffusion problems via complex variable reproducing kernel particle method. Chin Phys B 2013; 22(9)09:090204-1-6

[7] Yankelevsky D Z. Elastic-plastic behavior of a shallow two bar truss [J]. International Journal of Mechanical Sciences, 1999, 41 (6): 663-675

[8] Rackliffe M E., Jensen D W., Lucas W K. Local and global buckling of ultra-ligheweight Iso Truss structures [J]. Composites Science and Technology, 2006, 66 (2): 283-288

[9] Zhang LW, Liew KM. An element-free based solution for nonlinear Schro-dinger equations using the ICVMLS-Ritz method. Appl Math Comput 2014; 249: 333-45

[10] Guo Shujuan, Kong Xiangjun, Han Jie. Finite element analysis of tower crane $[\mathrm{J}]$. Science Technology and Engineering, 2010, 10 (1):204-206

[11] Sun Zhi, Hou Nin, Xiang Hai Fang. Safty and serviceability assessment for high-rise tower crane to turbulent winds [J]. Frontiers of Architecture and Civil Engineering, 2009, 3 (1): $18-24$
[12] Peng Miaojuan, Li Dongming, Cheng Yumin. The complex variable element-free Galerkin (CVEFG) method for elasto-plasticity problems. Engineering Structures, 2011, 33 (1): $127-135$

[13] Cheng Rongjun, Cheng Yumin. Error estimate of element-free Galerkin method for elasticity. Acta Physica Sinica, 2011, 60 (7): 070206

[14] Zhang LW, Lei ZX, Liew KM. Computation of vibration solution for functionally graded carbon nanotube-reinforced composite thick plates resting on elastic foundations using the element-free IMLS-Ritz method. ApplMath Comput 2015; 256: 488-504

[15] ABAQUS, 2008. Theory Manual, Version 6.7. Hibbit. Karlsson and Sorensen Inc., Pawtuchet, RI

[16] Li DM, Cheng YM, Liew KM. Animproved complex variable element-free Galerkin method for two-dimensional large deformation elastoplasticity problems. Comput Method Appl Mech Eng 2014; 269: 72-86

[17] Zeng Pan, Lei Liping. ANSYS, finite element analysis guide: modeling and analysis of structure [M]. Beijing: China Machine Press, 2010

[18] Giovanni Garcea, Antonio Madeo, Giuseppe Zagari, Raffaele Casciaro. Asymptotic post-buckling FEM analysis using corotational formulation,46 (2009) 377-397

[19] Cheng YM, Wang WQ, Peng MJ, Zhang Z. Mathematical aspects of meshless methods. Math Probl Eng 2014; 2014: $756297-1-4$

[20] Chen L, Cheng YM, Ma HP. The complex variable reproducing kernel particle method for the analysis of Kirchhoff plates. Comput Mech 2015; 55 (3): 591-602

[21] Pi, Y.-L., Bradford, M. A., 2012. Non-linear in-plane analysis and buckling of pinned fixed shallow arches subjected to a central concentrated load. International Journal of Non-Linear Mechanics 47 (1), 118-131

[22] Von Karman T., Tsien H S. The buckling of thin cylindrical shells under axial compression [J]. 2004, 303-312

[23] Bushnell D. Computerized buckling analysis of shells [M]. Dordrecht: Springer Netherlands. 2007

[24] Chen Tieyun., Bucking of structures [M]. Shanghai: Shanghai Science and Technology Press, 2011

[25] Abichou, H., Zahrouni, H., Potier-Ferry, M., 2002. Asymptotic numerical method for problems coupling several nonlinearities. Computer Methods in Applied Mechanics and Engineering 191 (51-52), 5795-5810

[26] Zhang LW, Liew KM. An improved moving least-squares Ritz method for two-dimensional elasticity problems. Appl Math Comput 2014; 246: 268-82

[27] Cheng YM, Bai FN, The interpolating element-free Galerkin (IEFG) method for two-dimensional elastoplasticity. Applmath model 2014; 38: 5187-97 